\section{Improved Reliability of Allele-Specific PCR}

BioTechniques 33:484-490 (September 2002)

Allele-specific PCR is considered to be the most straightforward approach for the detection of SNPs $(8,9)$. However, its application remains rather limited, mainly because of insufficient reliability. Indeed, under suboptimal conditions, allele-specific primers tend to amplify both matched and mismatched alleles, thus leading to an overestimation of the proportion of heterozygotes. Tremendous efforts have been undertaken to overcome this disadvantage. Conventional improvements include rigorous optimization (i.e., minimization) of the concentration of the key PCR components (primers, nucleotides, magnesium chloride, DNA polymerase, DNA template, etc.), careful adjustment of the annealing temperature and cycling conditions, introduction of additional destabilizing mismatches into the primers sequence, etc. (9). Importantly, all these modifications eventually aim to increase the stringency of PCR up to its limits, thus negatively affecting the total PCR yield. As a result, the tiny window of optimal conditions lies between nonspecific and unsuccessful amplification; hence, researchers often prefer to avoid allele-specific PCR if other, even more expensive and laborious options are possible (4). Recent developments in the real-time monitoring of allele-specific PCR have substantially improved its reliability $(3,8)$; however, even this kinetic format requires tedious optimization of reactions. Furthermore, the cost of highthroughput instrumentation and reagents is often incompatible with the budget of academic and medical diagnostic laboratories. Thus, the need for improvement of conventional approaches remains significant (3).

The best conditions for nonspecific mispriming occur in the very first cycles of allele-specific PCR, when the DNA template is subjected to an excess of PCR components. Once mismatched product has appeared, it undergoes perfectly efficient amplification because its polymorphic nucleotide already corresponds to the primer, not to the original DNA template. Hence, the specificity of priming during the initial rounds of PCR is vital for the success of the method. This accuracy can be achieved by very stringent PCR conditions; however, that excessive PCR stringency becomes undesirable in the later cycles when significant accumulation of the PCR product is expected. The ideal way to improve allele-specific PCR would involve the creation of a flexible deposit of a key PCR component so that the availability of the limiting PCR molecule is being adjusted to the growing amount of PCR template. Surprisingly, this goal can be achieved by a very simple option, namely the addition of the oligonucleotide complementary to the allele-specific primer (Figure 1).

Under conditions of the usual allelespecific PCR format, the priming efficiency depends on the rate of two processes, such as primer annealing and primer extension (11). Primer annealing is not a major limiting factor, as the excess of either matched or mismatched oligonucleotide ensures nearly constant engagement of the corresponding sequence in the DNA template. Thus, the specificity of allele-specific PCR mainly depends on the difference in primer extension for the matched versus mismatched nucleotides (Figure 1, left).
This difference itself may often be insufficient to discriminate between alternative alleles (1).

The PCR conditions change if the bulk of allele-specific primer is bound to the complementary oligonucleotide (Figure 1, right). The DNA template is no longer constantly occupied by the primer because the latter is diverted to the excess of competitor. However, since the duplexes in the annealing conditions are not stable and undergo repeated association/dissociation, the chance of temporary annealing to DNA template still remains. Further fate of the primer heavily depends on whether its $3^{\prime}$ nucleotide matches or mismatches. In the case of a match, even temporary hybridization to the DNA template is followed by immediate primer extension because of the residual activity of the Taq DNA polymerase at the annealing temperatures. Thus, the matched primer is extended and loses the ability to dissociate from the template. On the contrary, the extension of the $3^{\prime}$ mismatched primer is compromised, thus increasing its chances to dissociate from the DNA template before the elongation occurs. Noticeably, the association/dissociation between allele-specific primer and its corresponding complementary oligonu-

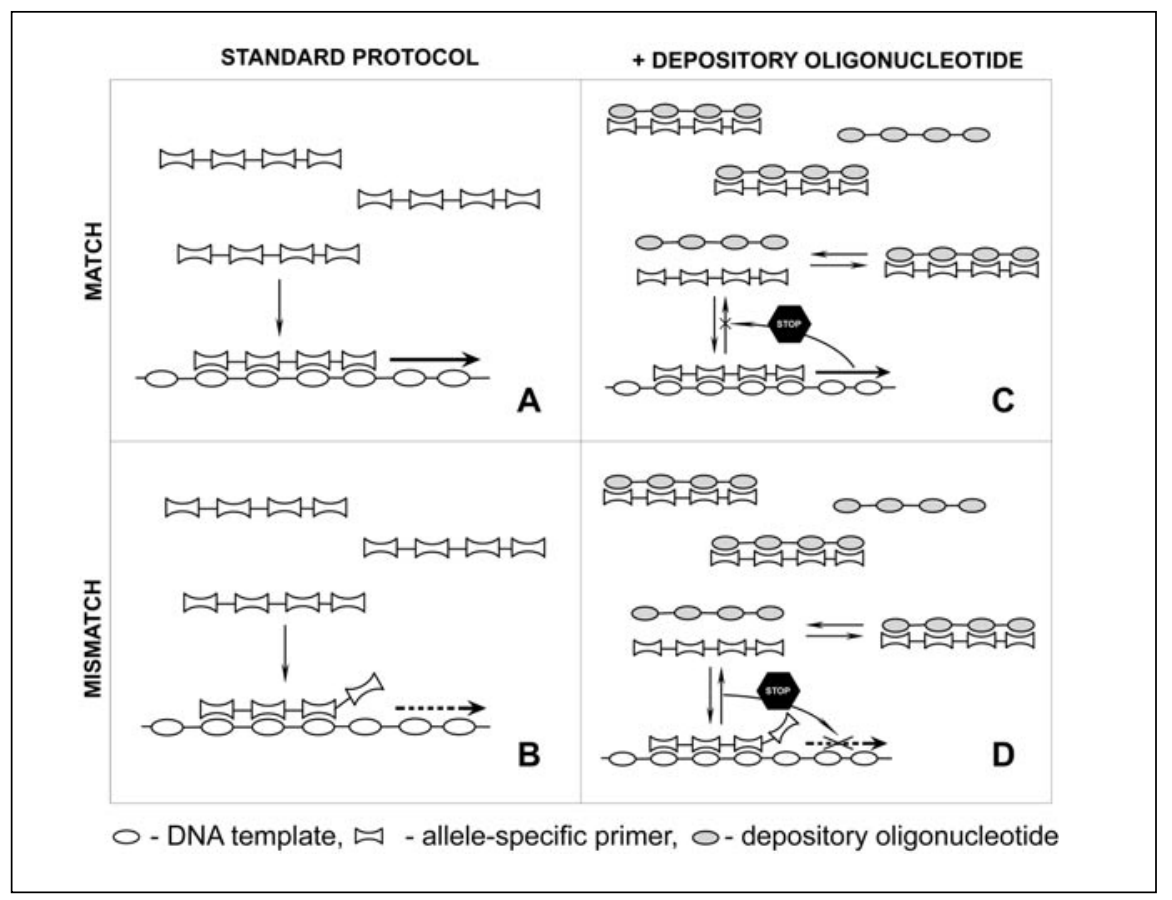

Figure 1. Schematic representation of conventional allele-specific PCR (left) and allele-specific PCR with reversible deposition of allele-specific primers by complementary oligonucleotides (right). 
cleotide is absolutely reversible because neither of the partners undergoes any modification. Therefore, the absolute amount of allele-specific primer remains sufficient to support effective DNA template amplification even in the later stages of PCR. Overall, the proposed design is expected to secure the specificity of allele-specific PCR over a broader range of conditions and without affecting the PCR yield.

To test this hypothesis, we selected the G/A polymorphism in $-308 \mathrm{nu}-$ cleotide of the TNF- $\alpha$ gene. The true genotypes were determined by PCRRFLP genotyping (5), which is unlikely to produce false results. The allele-specific PCR included two allele-specific primers [5'-ATAGGTTTTGAGGGGCATGG-3' (primer G) and 5'-ATAGGTTTTGAGGGGCATGA- $3^{\prime}$ (primer A); the variable nucleotide is underlined], and the common primer $5^{\prime}$-TCTCGGTTTCTTCTCCATCG-3' (10). In our hands, the conventional allele-specific PCR retained specificity only under very stringent conditions, which included a low concentration of primers $(0.125 \mu \mathrm{M})$ and magnesium chloride $(1.0 \mathrm{mM})$, a high annealing temperature $\left(60^{\circ} \mathrm{C}\right)$, and a low number of PCR cycles $(n=30)$ (Figure 2$)$. When any of the mentioned parameters was relaxed, the nonspecific reaction occurred (Figure 2, left column). On the contrary, increasing the PCR stringency led to the absence of the product (data not shown). Thus, the range of conditions for this allele-specific PCR was very narrow, and the reproducible genotyping could not be achieved.

Next, the same reactions were run in the presence of complementary depository oligonucleotides $\left(5^{\prime}\right.$-CCATGCCCCTCAAAACCTAT- $3^{\prime}$ for primer $\mathrm{G}$ and 5'-TCATGCCCCTCAAAACCTAT-3' for primer A; 3 -fold excess as compared to the concentration of allele-specific primers). With this modification, relaxation of any of the mentioned above critical parameters did not result in nonspecific amplification (Figure 2, right column). Moreover, allele-specific PCR retained the specificity even when several PCR variables were driven into suboptimal range simultaneously (Figure 2, lower panel). Thus, the deposition of allele-specific primers by complementary oligonucleotides evidently in- creased the reliability of the method.

The degree of excess of the "depository" oligonucleotides turned out not to be critical. Under moderately stringent conditions, significant improvement of allele-specific PCR specificity was already achieved with the 1:1 ratio, and the reactions remained successful even with the 5- to 10-fold excess of the competitor (data not shown). Perfect matching between the allele-specific primer and the complementary oligonucleotide also was not a prerequisite, as essentially the same results were obtained both with corresponding and mismatched primer/competitor pairs (data not shown). The latter observation is expected because a single mismatch in the terminating nucleotide is unlikely to affect considerably the kinetics of association/dissociation of 20-mer duplexes, especially if one of the partners is present in excess. This fact substantially in-

creases the flexibility of the suggested assay: the synthesis of just one of the depository oligonucleotides should be sufficient for both allele-specific reactions, and the choice of the terminating nucleotide could be determined not by match/mismatch considerations, but rather by avoiding the undesirable side effects (formation of primer dimers, secondary structures, etc.). At least in theory, this "universal" competitor may simply lack the counterpart of the variable base, thus being somewhat shorter than the allele-specific primers.

The present approach has something in common with so-called "competitive" variations of allele-specific PCR (2,6-8). In particular, several investigators suggested adding both allele-specific primers in the same tube so that the matched and mismatched oligonucleotides would compete with each other $(2,8)$. However, in this case, the ratio

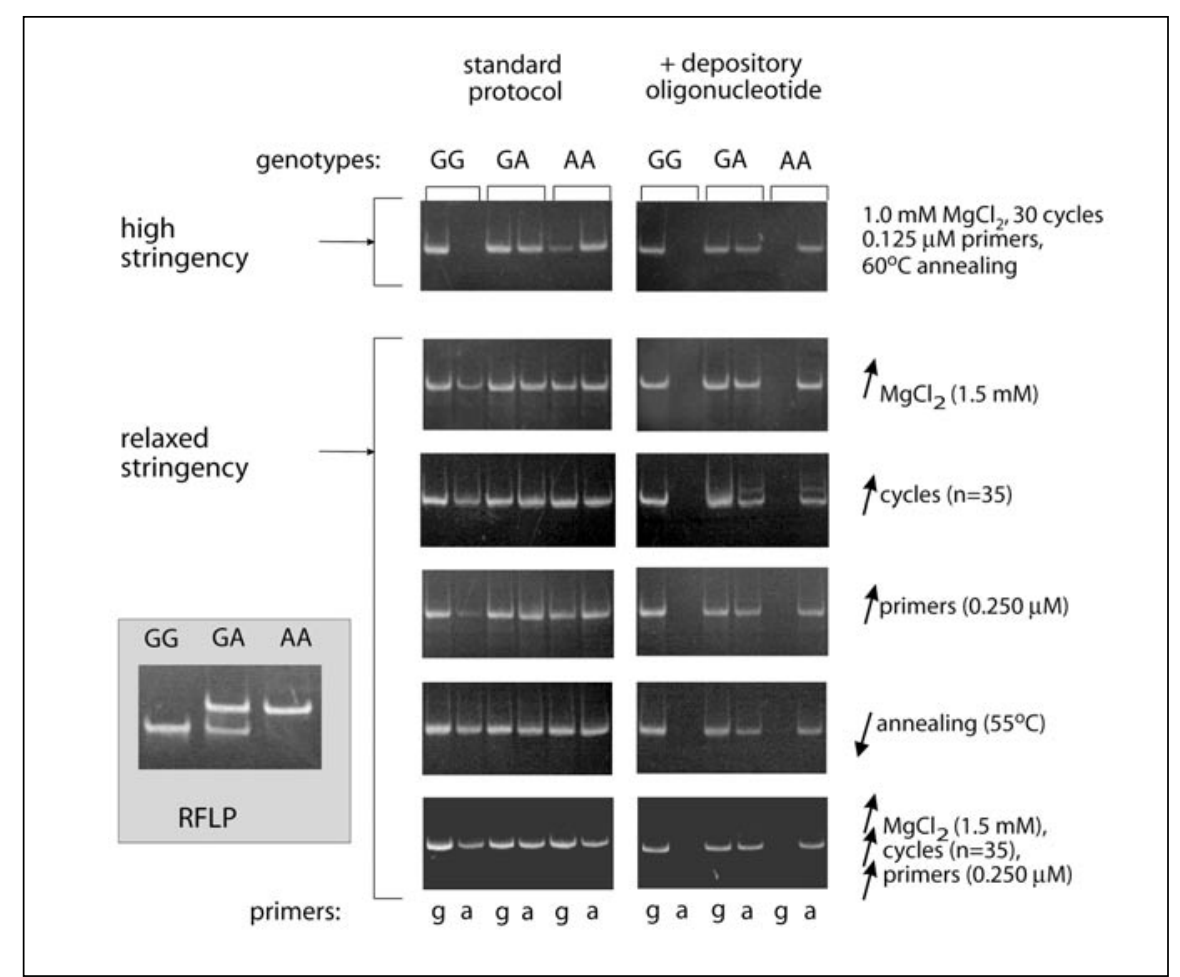

Figure 2. Deposition of allele-specific primers by complementary oligonucleotides broadens the range of conditions for allele-specific PCR. Representative homozygous and heterozygous genotypes of the TNF- $\alpha$ gene were amplified by the conventional allele-specific PCR protocol (left column), or in the presence of a 3-fold excess of the corresponding depository oligonucleotide (right column). The original reactions (upper panel) included $50 \mathrm{ng}$ genomic DNA, 0.5 U heat-activated Taq DNA polymerase, 10 $\mathrm{mM}$ Tris- $\mathrm{HCl}$ (pH 8.3), $50 \mathrm{mM} \mathrm{KCl}, 5 \%$ glycerol, $1.0 \mathrm{mM} \mathrm{MgCl}_{2}, 200 \mu \mathrm{M}$ dNTP, $0.125 \mu \mathrm{M}$ each primer in a final volume of $10 \mu \mathrm{L}$ and were carried out for 30 cycles of $95^{\circ} \mathrm{C}$ for $35 \mathrm{~s}, 60^{\circ} \mathrm{C}$ for $60 \mathrm{~s}$, and $72^{\circ} \mathrm{C}$ for $50 \mathrm{~s}$ after an initial activation of the polymerase at $95^{\circ} \mathrm{C}$ for $10 \mathrm{~min}$. Upon subsequent relaxation of the PCR conditions (lower panels; see details at the right), conventional allele-specific PCR failed to determine the genotypes correctly, whereas the modified protocol retained the specificity. See also the example of RFLP genotyping of the same DNA samples. 
between "competitor" and allele-specific primer approaches only $1: 1$, which may be insufficient in certain situations (1). Furthermore, the allele-specific products should be distinguished (e.g., by a difference in length or fluorescent color), which requires additional effort $(2,8)$. A potentially more powerful solution involves the introduction into the sequence of competitor non-extendable 3 '-end nucleotide (e.g., dideoxy-NTP) (6). However, in the first rounds of PCR, such a "competitive blocker" may fully occupy minute amounts of specific DNA template, thus significantly reducing the PCR yield even for the specific allele. This puts some limitations on the ratio among competitive blocker, specific primers, and target DNA sequence $(6,7)$. Another disadvantage is attributed to purely technical reasons: the production of competitive blockers is not routine for most manufacturers, so the addition of the ddNTP to the $3^{\prime}$ end of the primer is often done by researchers themselves using expensive, troublesome, and difficult-to-control enzymatic synthesis (7). Contrary to the methods described above, our modification appears to be a very simple procedure. Moreover, it does not affect the availability of the relatively underrepresented original DNA template. On the contrary, it creates a reversible, specific depository for the excessive component of the PCR amplification, namely for allele-specific primers. Therefore, the reaction remains favorable for both specificity and sensitivity over a relatively wide range of conditions.

In summary, this report demonstrates that the deposition of allele-specific primers by an excess of complementary oligonucleotides evidently increases the reliability of allele-specific PCR. In contrast to previously described procedures $(8,9)$, the suggested protocol of allele-specific PCR does not require an extensive optimization of the reaction conditions. Notably, the concentration of complementary oligonucleotides may vary within a significant range without affecting the success of allele discrimination. Further experiments are required to determine how the peculiarities of each particular genotyping assay (i.e., nucleotide composition of polymorphic sequence, primers length, etc.) would affect the suitability of this tech- nique. If proven to be widely applicable, then the proposed modification may substantially facilitate SNP genotyping, either alone or in combination with other allele-specific PCR improvements. Its contribution could be especially valuable for the conventional allele-specific PCR protocol, which includes double-tube allele-specific amplification followed by gel visualization of the product. Furthermore, the addition of complementary depository oligonucleotides may also simplify the optimization of the modern high-throughput methods of direct allele discrimination.

\section{REFERENCES}

1.Ayyadevara, S., J.J. Thaden, and R.J. Shmookler Reis. 2000. Discrimination of primer 3'-nucleotide mismatch by Taq DNA polymerase during polymerase chain reaction. Anal. Biochem. 284:11-18.

2.Dutton, C. and S.S. Sommer. 1991. Simultaneous detection of multiple single-base alleles at a polymorphic site. BioTechniques 11:700702.

3.Kristensen, V.N., D. Kelefiotis, T. Kristensen, and A.L. Borresen-Dale. 2001 High-throughput methods for detection of genetic variation. BioTechniques 30:318-332.

4.Malcolm, E.K., G.B. Baber, J.C. Boyd, and M.H. Stoler. 2000. Polymorphism at codon 72 of p53 is not associated with cervical cancer risk. Mod. Pathol. 13:373-378.

5.Mestiri, S., N. Bouaouina, S.B. Ahmed, A. Khedhaier, B.B. Jrad, S. Remadi, and L. Chouchane. 2001. Genetic variation in the tumor necrosis factor- $\alpha$ promoter region and in the stress protein hsp70-2: susceptibility and prognostic implications in breast carcinoma. Cancer 91:672-678.

6.Orou, A., B. Fechner, G. Utermann, and H.J. Menzel. 1995. Allele-specific competitive blocker PCR: a one-step method with applicability to pool screening. Hum. Mutat. 6:163-169.

7.Parsons, B.L. and R.H. Heflich. 1998. Detection of a mouse H-ras codon 61 mutation using a modified allele-specific competitive blocker PCR genotypic selection method. Mutagenesis 13:581-588.

8.Ravine, D. 1999. Automated mutation analysis. J. Inherit. Metab. Dis. 22:503-518.

9.Sommer, S.S., A.R. Groszbach, and C.D. Bottema. 1992. PCR amplification of specific alleles (PASA) is a general method for rapidly detecting known single-base changes. BioTechniques 12:82-87.

10.Verjans, G.M., B.M. Brinkman, C.E. Van Doornik, A. Kijlstra, and C.L. Verweij. 1994. Polymorphism of tumour necrosis factor- $\alpha(\mathrm{TNF}-\alpha)$ at position -308 in relation to ankylosing spondylitis. Clin. Exp. Immunol. 97:45-47.

11.Wu, D.Y., L. Ugozzoli, B.K. Pal, and R.B. Wallace. 1989. Allele-specific enzymatic am- 
plification of $\beta$-globin genomic DNA for diagnosis of sickle cell anemia. Proc. Natl. Acad. Sci. USA 86:2757-2760.

This work is supported by INTAS grant no. 99-01391, INCO-Copernicus grant no. ICA2-CT-2000-10056, and RFBR grant no. 02-04-49890. Address correspondence to Dr. Evgeny Imyanitov, Group of Molecular Diagnostics, N.N. Petrov Institute of Oncology, St.-Petersburg 197758, Russia. e-mail: evgeny@imyanitov.spb.ru

Received 24 May 2002; accepted 2 July 2002.

E.N. Imyanitov, K.G. Buslov, E.N. Suspitsin, E.Sh. Kuligina, E.V. Belogubova, M.Yu. Grigoriev, A.V. Togo, and K.P. Hanson

N.N. Petrov Institute of

Oncology

St.-Petersburg, Russia

For reprints of this or any other article, contact Reprints@BioTechniques.com

\section{Construction of a Broad Host Range Cloning Vector Conferring Triclosan Resistance}

BioTechniques 33:490-492 (September 2002)

Triclosan is an antimicrobial found in deodorants, toothpaste, hand lotions, and acne treatments, along with many other household products and is an effective inhibitor of both Gram-positive and Gram-negative species (8). Recently, there has been increased research into the mechanisms whereby bacteria become resistant to the antimicrobial effects of triclosan. McMurry et al. $(10,11)$ identified the enoyl-acyl carrier protein (ACP) reductase, FabI, as the main intracellular enzyme target of triclosan in E. coli. It has also been shown, for example, that some bacteria contain orthologous enoyl-ACP reductases, namely FabL and FabK, which are not inhibited by triclosan $(6,7)$. Bacteria that contain these alternate enzymes are able to tolerate higher concentrations of triclosan than those bacteria that have only the FabI-type enoyl-ACP reductase $(6,7)$.

The construction of a vector with a triclosan resistance determinant would make triclosan a useful addition to the selection agents that are currently available. Triclosan is effective against a range of bacteria at less than $10 \mathrm{ppm}$ (8). In E. coli, even mutants with increased resistance are still inhibited at approximately $10 \mathrm{ppm}$ triclosan (10, 11). Triclosan is also nontoxic to humans $(3,8)$. These characteristics make triclosan an attractive selective agent. However, no cloning vectors are currently available with triclosan resistance determinants. Here we report the construction of a broad host range plasmid that is amenable to blue-white screening and that contains a triclosan resistance marker.

The enoyl-ACP reductase gene $f a b L$ (6), including the promoter, was amplified by PCR from Bacillus subtilis chromosomal DNA using the primers YgaABam (5'-GGATCCTTAAACGAGCAGTGAGCGTCCGCCGTC-3') (6) and YgaABam 3 (5'-GGATCCCCCTTTAAAGTTCTTGCCA-3'). Both primers were designed with BamHI sites (in bold) on the $5^{\prime}$ ends. PCR product was ligated into pGEM-T $^{\circledR}$ Easy (Promega, Madison, WI, USA) and transformed into One-Shot ${ }^{\mathrm{TM}}$ (Invitrogen, Carlsbad, CA, USA) chemically competent cells following the manufacturers' instructions. Clones containing the $f a b L$ insert were identified through gel electrophoresis of BamHI-digested plasmid. The $f a b L$ fragment was purified from the agarose gel and ligated into $B g l I I-d i g e s t e d$ pBBR1-MCS2 (9). The ligation reaction was electroporated into $E$. coli $\mathrm{S} 17$ cells, and transformants were selected on LB agar containing 50 ppm kanamycin and $10 \mathrm{ppm}$ triclosan. Several transformants were obtained, and three were analyzed by restriction digest with HindIII. All contained fabL in the same orientation. This new vector was called pBBRT (Figure 1).

E. coli $\mathrm{S} 17$ containing pBBRT was streaked on LB containing up to 10000 ppm triclosan to determine the level of resistance. Also, growth curves of $E$. coli S17 with or without pBBRT were determined in liquid LB medium at varied concentrations of triclosan. In a 96well microplate, overnight culture of either S17 or S17 pBBRT was inoculated into LB containing 0-100 ppm triclosan. Absorbance at $600 \mathrm{~nm}$ was measured over time in a MicroQuant ${ }^{\mathrm{TM}}$ (Bio-Tek ${ }^{\circledR}$ Instruments, Winooski, VT, USA) spectrophotometer with $\mathrm{KC} \mathrm{Ju}$ nior $^{\mathrm{TM}}$ software (Bio-Tek Instruments).

E. coli $\mathrm{S} 17 \mathrm{pBBRT}$ was able to grow in liquid media containing up to 25 ppm triclosan. At $50 \mathrm{ppm}$ and higher, growth of S17 pBBRT was slow, although not completely inhibited (data not shown). The parental E. coli S17 strain has a minimum inhibitory concentration of less than $1 \mathrm{ppm}$ (data not shown). On LB agar plates, however, S17 pBBRT was able to grow in the presence of up to $10000 \mathrm{ppm}$ triclosan, whereas the parent strain could not grow on plates containing $2 \mathrm{ppm}$ triclosan. The tolerance of higher triclosan levels by S17 pBBRT on the plate may be due to diffusional constraints of the excess triclosan, as triclosan is only soluble to approximately $10 \mathrm{ppm}$ in water (4). To demonstrate the utility of this construct in other organisms, pBBRT was also transferred 